\title{
INTERNATIONAL SYSTEM OF ELECTRIC AND MAGNETIC UNITS
}

By J. H. Dellinger

CONTENTS

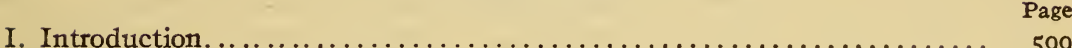

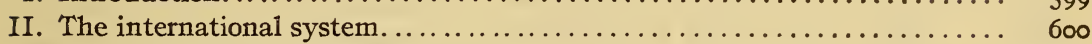

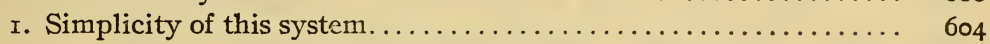

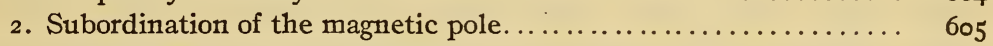

3. Limitation to electromagnetism...................... 605

III. The magnetic quantities.................................. 606

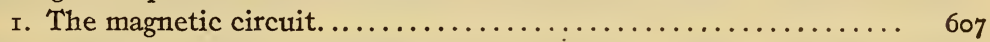

2. Induction and magnetizing force..................... 608

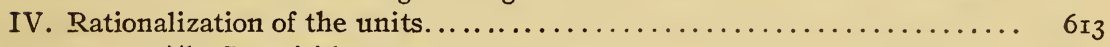

I. The Heaviside system.............................. $6 \mathrm{r}_{3}$

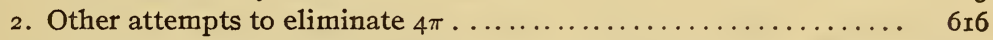

3. The two sets of magnetic units...................... $62 \mathrm{r}$

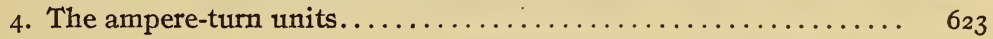

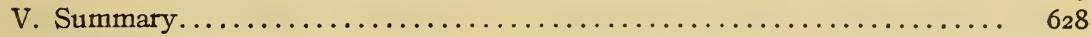

Appendix.-Symbols used..................................... $6_{3}$ I

\section{INTRODUCTION}

Electric units and standards are now very nearly uniform in all parts of the world. As a result of the decisions of international congresses and the cooperation of the national standardizing laboratories, the international ohm and ampere are accepted everywhere as the basis of electrical measurements. A complete system of electric and magnetic units is in general use, built upon these fundamental units. There have been proposals to change the units from time to time, and as a result there is some diversity of usage in respect to units in current electric and magnetic literature. For instance, Heaviside units are used in certain recent books on theoretical electricity; a slight change of electric units is advocated by $M$. Abraham; and quite a number of different magnetic units are used in presenting the results of magnetic experiments. It appears worth while to examine critically the system of units which is generally used, to study the reasons which are advanced 
for changes in the system, and to inquire whether any of the various proposed units offer advantages which would justify the trouble and confusion incident to a general change of units.

The units actually used in electrical measurements are based on certain fundamental electrical standards which were so defined as to represent, as closely as experimental evidence permitted, the theoretical electromagnetic units. The units of the various electric quantities are derived from the fundamental units in accordance with the equations of ordinary electrical theory, and in consequence the units actually used in practice are all very close representatives of the corresponding electromagnetic units. The electromagnetic system is familiar, having been frequently described. The units used in practice to represent the electromagnetic units have been called the international units. The very slight differences in magnitude between the international and the corresponding electromagnetic units are determined by absolute measurements made from time to time. (The present values of these differences are given, on the basis of recent absolute measurements, in this Bureau's Circular No. 6o, Electric Units and Standards.) The system of international units will be discussed here in some detail before treating the various proposals which have been made for changes in the units. The said proposals would apply both to the electromagnetic units and to the international units, their representatives.

\section{THE INTERNATIONAL SYSTEM}

Every complete system of electric units requires four fundamental units instead of only three, as in mechanics. The necessity for the one additional unit arises from the fact that the identity of mechanical and electrical phenomena has not been established. Of the four quantities taken as fundamental (a) three may be mechanical and one electrical, or (b) two mechanical and two electrical, or $(c)$ one mechanical and three electrical, the only theoretical requirement being that both mechanical and electrical quantities be represented among the four. Thus, very many systems are possible. No systems of type (c) are in use, although they can be very easily made up, and are not at all grotesque-for example, a system using $R, I, \mu$, and $T$ as fundamental.

The electrostatic and electromagnetic systems are of type (a). The fundamental units in each of these systems are those of length, mass, time, and a property of the eletromagnetic medium. The property of the medium utilized is the dielectric constant in the 
electrostatic system and magnetic permeability in the electromagnetic system. For convenience, zero dimensions are sometimes assigned to the property of the medium in writing dimensional formulas, but it remains none the less a fundamental quantity of the system.

The international system is of type $(b)$. In the definitions adopted by the London Electrical Conference of 1908 two electric units were defined in terms of specified standards-the international ohm and the international ampere. These two, plus units of length and time, may be regarded as forming the basis of a distinct system, and they have been so considered by a few writers. This system is very convenient in electrical work, although it has no value or utility in mechanics or other parts of physics. The system is centered around the phenomena of electric current. Electric current is more familiar and of vastly greater practical importance than electrostatic charges or magnetic poles, upon which the other two familiar systems are based. This is one of the reasons why the international system is the most convenient and the most used electrical system.

Only eight units (the international ohm, ampere, volt, coulomb, henry, farad, joule, and watt) have been defined by international congresses, and it is desired to show that these units form part of a distinct and complete electrical system. That the international units form a system distinct from all others may be seen from the following considerations. The international units are historically based upon the "practical" electromagnetic units, as has been stated above. However, two of the international electric units are defined in terms of arbitrary standards and hence are independent or arbitrary ${ }^{1}$ units, viz, the international ohm and the international ampere. Since a complete system of electric units requires four independent units, only two additional units are needed. Therefore the international system can not have all four of the fundamental quantities of the electromagnetic system, viz, length, mass, time, and magnetic permeability. The two of these quantities which are needed to complete the international system are length and time. Both length and time enter essentially in

1 The international ohm and ampere are independent units in spite of the fact that in the realization of the standards by means of which they are defined other physical quantities are utilized. The international ohm is defined as the resistance of a uniform column of mercury of specified length and mass. This length and mass, however, are merely auxiliary to the preparation of the standard. The unit is the actual resistance of such a standard, and its magnitude would not vary if different units of length and mass were used in the definition of the standards. The case is very similar to the definition of the unit of temperature on the international hydrogen scale. This is strictly an arbitrary unit, and yet in its definition (see this Bulletin 3, p. 664; I907) use is made of the meter to specify the initial pressure in the hydrogen thermometer. 
the fundamental relations between the important electromagnetic quantities. (This is clearly exhibited in Table I below.) Mass does not enter at all into those relations, and magnetic permeability is a subordinate quantity in this system just as it is in the electrostatic system.

That the international units form a complete electrical system, which is in fact the system commonly used, is brought out by Table $I$, in which the units of the principal electric and magnetic quantities are derived from the units of resistance, current, length, and time by the use of the ordinary equations of electrical theory. The international volt, coulomb, etc., were thus derived from the international ohm and ampere and the second in the definitions adopted by international congresses. Each unit in this system is the representative for practical purposes of the corresponding unit in the electromagnetic system and differs from it only very slightly. The small differences between the units in the two systems are determined by absolute measurements made from time to time.

This mode of definition was thus extended to the whole system of electric and magnetic units by Prof. V. Karapetoff in the appendixes of his books, The Electric Circuit (I9I2) and The Magnetic Circuit (I9II). He calls the units so obtained the "ampereohm system." In his derivations, however, he uses some equations differing by the factor $4 \pi$ from the classical equations. Certain of the units thus derived are therefore not representatives of the corresponding electromagnetic units. Consequently his ampere-ohm system is not quite the same as the "international system" as interpreted herein. The Karapetoff units are discussed on page 620 below.

The table gives the dimensional formula for each quantity, as well as the defining equation or equation which gives the mathematical relation of the quantity to previously defined quantities. All the symbols used are defined in the table, except the following, which are introduced without definition: $L=$ length, $T=$ time, $R=$ resistance, $I$ = current, $N=$ number of turns, $S=$ area, $s=$ length along a path. 
TABLE 1

The International System

\begin{tabular}{|c|c|c|c|c|}
\hline Quantity & Symbol & Unit $a$ & Defining equation & $\begin{array}{l}\text { Dimensional for- } \\
\text { mula }\end{array}$ \\
\hline Electromotive force..... & E & Volt... & $E=R I \ldots$ & {$[E]=[R I]$} \\
\hline Quantity of electricity.. & $Q$ & Coulomb. & $Q=\int I d t \ldots$ & {$[Q]=[I T]$} \\
\hline Electrostatic capacity... & $C$ & Farad.... & $C=\frac{Q}{E} \ldots \ldots$ & {$[C]=\left[\frac{T}{R}\right]$} \\
\hline Dielectric constant..... & K & & $\begin{aligned} C= & \frac{K S}{4 \pi l}(\text { for a par- } \\
& \text { allel plate con- } \\
& \text { denser of large } \\
& \text { area } S) .\end{aligned}$ & {$[K]=\left[\frac{T}{L R}\right]$} \\
\hline Electric field intensity.. & ह & Volt per $\mathrm{cm} .$. & $\varepsilon=\frac{d E}{d s} \ldots$ & {$[E]=\left[\frac{R I}{L}\right]$} \\
\hline Electric displacement.. & $D$ & $\begin{array}{l}\text { Coulomb per } \\
\mathrm{cm}^{2} .\end{array}$ & $D=\frac{K}{4 \pi}$ こ. & {$[D]=\left[\frac{I T}{L^{2}}\right]$} \\
\hline Self-inductance $b$...... & $\mathcal{L}$ & Henry...... & $\mathcal{L}=\frac{L_{1}}{d I_{1} / d t} \ldots$ & \\
\hline Mutual inductance $c \ldots$ & $m$ & Henry... & $m=\frac{E_{2}}{d I_{1} / d t} \cdots$ & \\
\hline Magnetomotive force... & F & See Table 6. & $\mathcal{F}=\frac{4 \pi}{10} N I \ldots$. & {$[\mathfrak{F}]=[I]$} \\
\hline Magnetizing force $d . \ldots$ & $H$ & ....do.. & $H=\frac{4 \pi N I}{10 L}$ & {$[H]=\left[\frac{I}{L}\right]$} \\
\hline Magnetic flux $e \ldots . . .$. & $\phi$ & .....do... & $\phi=10^{8} \int_{0}^{t} E d t \ldots$ & {$[\phi]=[R I T]$} \\
\hline Magnetic induction..... & B & .....do.. & $B=\frac{d \phi}{d S} \ldots \ldots$ & {$[B]=\left[\frac{R I T}{L^{2}}\right.$} \\
\hline Permeability...... & $\mu$ & ....do.. & $\mu=\frac{B}{H}$. & {$[\mu]=\left[\frac{R T}{L}\right]$} \\
\hline Reluctance. . & R & ....do. & $R=\frac{F}{\phi} \ldots \ldots$. & {$[R]=\left[\frac{1}{R T}\right]$} \\
\hline $\begin{array}{l}\text { Intensity of magnetiza- } \\
\text { tion. }\end{array}$ & $J$ & .....do.. & $B=B_{0}+4 \pi J$ & {$[J]=\left[\frac{R I T}{L^{2}}\right]$} \\
\hline Magnetic susceptibility. & $\kappa$ & ....do. & $J=\kappa H$ & {$[\kappa]=\left[\frac{R T}{L}\right]$} \\
\hline Magnetic pole strength $f$. & $m$ & .....do... & $\phi=4 \pi m$ & {$[m]=[R I T]$} \\
\hline
\end{tabular}

$a$ The names of these units are strictly the "international volt," "international coulomb," etc., but in practice "international" is omitted.

$b$ In the definition of self-inductance, $E_{1}$ is the emf created in a circuit by the variation of the current $I_{1}$ in the same circuit.

$c$ In the definition of mutual inductance, $E_{2}$ is the emf induced in a circuit by the variation of the current $I_{1}$ in another circuit.

d $H$ in the defining equation is the magnetizing force at the center of a very long solenoid of $N$ turns and length $L$.

$e \phi$ in the equation is the change of flux in the time $t$ giving rise to the emf $E$ in a closed circuit linking with the flux.

$f \phi$ in this equation is the total magnetic flux emanating from or associated with magnetic poles of aggregate pole strength $m$; it is strictly equal to the resultant flux through the magnet only when the magnet is very long and thin. 


\section{SIMPLICITY OF THIS SYSTEM}

We build up our electrical equations and make measurements in terms of resistance, current, length, and time. It is entirely legitimate to write dimensional expressions in terms of these quantities. The dimensional formulas in the international system of electric units, in fact, give expressions which are as simple and as helpful in electricity as the usual dimensional expressions in length, mass, and time are for mechanical quantities. This advantage is decidedly lacking in the electrostatic and electromagnetic systems. The superiority of the international system in this respect is clearly shown by an example. The dimensional formula for magnetic field intensity or magnetizing force is

$$
[H]=\left[\frac{I}{L}\right]
$$

This gives as clear and simple a statement of the relations of the two electromagnetic quantities, $H$ and $I$, as the dimensional formula for force, below, gives for mechanical quantities. Compare with the dimensional formula for $H$ in the electromagnetic system:

$$
[H]=\left[\frac{M^{1 / 2}}{L^{1 / 2} T \mu^{3 / 2}}\right]
$$

This is not convenient as regards the conveying of physical information. The point is that it bears no direct relation to the actual working equations of electricity. In the ordinary systems of mechanical units, the dimensional equations are almost the same as the defining equations of mechanical units. Thus the dimensional equation of force, $[F]=\left[\frac{M L}{T^{2}}\right]$, is similar to $F=M \frac{d^{2} L}{d t^{2}}$. Similarly, in the international system of electric units the dimensional equations are similar to the defining equations, which are the common equations of practice-for example, $[H]=\left[\frac{I}{L}\right]$ is closely related to $H=\frac{2 I}{r}$, which holds for an infinitely long, straight conductor.

The various dimensional formulas in the table very simply suggest the ordinary equations of electrical theory and practice. Take, for instance, $[D]=\left[\frac{I T}{L^{2}}\right]$. The functioning of electric dis- 
placement is at once apparent, for since $[I T]=[Q]$, it is suggested that electric displacement is quantity per unit area. The formulas $[C]=\left[\frac{T}{R}\right]$ and $[\mathcal{L}]=[R T]$ are very closely related to the expression for impedance

$$
Z=\sqrt{R^{2}+\left(\frac{2 \pi \mathcal{L}}{T}-\frac{T}{2 \pi C}\right)^{2}}
$$

where $T$ is the period of alternation. The dimensional formulas for $K$ and $\mu$ are both closely related to actual methods of measurement. $K$ depends on capacity $\left[\frac{T}{R}\right]$ and linear dimensions $[L] . \quad \mu$ may be determined by the measurement of a self-inductance $[R T]$ and linear dimensions $[L]$ of a coil. It of course follows from $[K]=\left[\frac{T}{L R}\right]$ and $[\mu]=\left[\frac{R T}{L}\right]$ that $\left[\frac{\mathrm{I}}{K \mu}\right]=\left[\frac{L^{2}}{T^{2}}\right]$, the familiar equation suggesting the influence of $K$ and $\mu$ upon the velocity of wave propagation.

\section{SUBORDINATION OF THE MAGNETIC POLE}

One of the advantages of the international system is that it does not give undue prominence to magnetic pole strength. The complexity of the dimensional expressions of the electromagnetic system and its poor correspondence to the conditions of practice are in part due to its being based upon an unimportant phenomenon. Magnetic poles are of little theoretical or practical importance, while magnetic flux, field intensity, etc., are. The medium is the important thing, as shown by Faraday and Maxwell. Magnetism is becoming more and more regarded as one kind of manifestation of electricity. In the table of the international system given above, magnetic pole strength was defined last, showing that all the other magnetic quantities are definable independently of it. (It can be similarly subordinated in the equations of the electrostatic system.) A free magnetic pole does not exist in nature, magnetic pole strength does not appear in engineering formulas, and it is consequently a satisfaction to find that it can be dispensed with in the theory also.

\section{LIMITATION TO ELECTROMAGNETISM}

The international electrical system of dimensional equations is not suitable as a basis for all physical quantities. It gives dimensions as awkward for the quantities of mechanics as the electro- 
magnetic system gives for electrical quantities, and is therefore not of such general application as the systems in which length, mass, and time are fundamental. The dimensions of mechanical quantities in this sytem are readily obtained as follows: Energy is the quantity common to electricity and mechanics, and it has the simple dimensions $\left[R I^{2} T\right]$. From the relation $\left[\frac{M L^{2}}{T^{2}}\right]=\left[R I^{2} T\right]$, the dimensions of mass and other mechanical quantities may be derived. Such dimensional expressions of mechanical quantities have no convenience or value. Furthermore, the unit of mass comes out $10^{7} \mathrm{~g}$ and the unit of force $10^{7}$ dynes, similarly to the unit of energy which is $10^{7}$ ergs (I joule). The international electrical system is valuable simply as a practical convenience in dealing with electrical phenomena. It can not be thought of as supplanting the classical system based on length, mass, and time, since the international ohm and ampere are not as fundamentally important as the kilogram and meter.

\section{THE MAGNETIC QUANTITIES}

The electrostatic and electromagnetic systems are primarily systems of physical quantities rather than units, and upon them various systems of units are based. Thus, for instance, both the cgs and the "practical" electromagnei systems of units are in use. These differ in the particular unics chosen for the fundamental quantities. In the case of the international electric units, only one particular set of units is used for the fundamental quantities; the four fundamental units are the international ohm, the international ampere, the centimeter, and the second. The defining equations given in Table $\mathrm{I}$, above, for $E, Q, C, \mathcal{L}$ and $\eta n$, are those by which the international units of these quantities were derived by international congresses. The only electric unit defined by the equations of Table $I$ that is not in use is the unit of dielectric constant. This would be expected, as the corresponding electromagnetic unit is never used, the electrostatic unit being universally employed. With this exception, the particular units indicated in Table I are the ones most commonly used. The units of the electric quantities correspond in magnitude to the "practical" electromagnetic units, and the magnetic units derived by the equations given in the table correspond to the cgs electromagnetic units. In addition to these magnetic units certain others are widely used. The latter units are the ampere-turn and units related to 
it. These units may be shown to constitute a complete alternative set of magnetic units, their use requiring certain of the equations to be changed by the factor $4 \pi$. The two sets of magnetic units are compared in Table 6 below.

Magnetic units involving the inch as the unit of length are also in use, particularly when the results of measurements are presented in the form of curves. While it would be possible to build up a definite system using the inch as the unit of length, these units are usually regarded as isolated units. When used in equations, they are accompanied by suitable numerical factors.

The nomenclature of the magnetic units is somewhat unsatisfactory. For instance, in the first set of magnetic units just referred to, at the present time the name "gauss" is applied both to the unit of induction and the unit of magnetizing force. In order to bring out clearly the difference between these two quantities, some general considerations regarding the magnetic circuit will be given here.

\section{THE MAGNETIC CIRCUIT}

A tube of magnetic induction is analogous to a tube of electric displacement, and also to a path along which electric current flows. The analogy is very close except that permeability and reluctance are not constant in magnetic material. The strictness of the analogies between electric and magnetic quantities is shown by the following table:

TABLE 2

\section{Comparison of Magnetic and Electric Quantities}

[See appendix at the end of this paper for symbols]

\begin{tabular}{|c|c|c|}
\hline & $\underset{\text { circuit }}{\operatorname{In} \text { a }}$ & $\begin{array}{c}\text { At a } \\
\text { point in } \\
\text { a medium }\end{array}$ \\
\hline 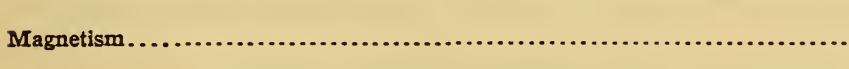 & $\phi=\frac{\tilde{F}}{R}$ & $B=\mu H$ \\
\hline Electric current... & $I=\frac{E}{R}$ & $U=\gamma \varepsilon$ \\
\hline Electrostatics........................................ & $Q=C E$ & $D=\frac{K}{4 \pi} \varepsilon$ \\
\hline
\end{tabular}

The analogy between the magnetic and the electrostatic relations is much better than that between magnetism and electric current in fact although not in form. There is no phenomenon of magnetic conduction corresponding to electric conduction, whereas magnetic induction and electric displacement are similar phenomena subject to the same laws. Reluctance is $75741^{\circ}-17-7$ 
strictly analogous to the reciprocal of capacity; this is suggested in the name elastance, which Heaviside gave to the reciprocal of capacity. The first and third equations in the last column are similar except for the factor $4 \pi$, which is without physical significance; in fact, this dissimilarity is merely a peculiarity of one set of magnetic units.

The only vector quantities in the table are $B$ and $H$ and their analogues, $D$ and $\mathcal{E}$ and $U$ and $\varepsilon$. Magnetomotive force $\mathcal{F}$ and electromotive force $E$ are line integrals of the two field intensities, $H$ and $\varepsilon$, respectively.

The term "magnetomotive force" (mmf) has been restricted by some writers to mean the total line integral of $H$ along a closed path. It would thus have a value different from zero only when the path of integration links with electric current. The line integral of $H$ along a part only of a closed path was called magnetic potential difference (mpd) or magnetic potential drop, or even magnetomotive force drop. According to these conventions, while a magnetic potential difference exists between the poles of a permanent magnet, yet one could not speak of a magnetomotive force due to the magnet, because the mpd between the poles in air is equal and opposite to the mpd within the magnet. If the term "electromotive force" were similarly restricted, there could be no emf due to a battery or a charged condenser.

Magnetomotive force is in fact the exact analog of electromotive force and bears the same relation to magnetic potential difference as emf does to electric potential difference. The potential difference between two points is the work done in carrying unit charge from one point to the other. The potential difference between the ends of any portion of a circuit is equal to the difference between the emf (or $\mathrm{mmf}$ ) produced in that portion, by any causes whatever, and the emf (or mmf) expended in that portion.

\section{INDUCTION AND MAGNETIZING FORCE}

It is common practice in electrical engineering to use the name "gauss" both for the unit of magnetic induction and the unit of magnetizing force. This usage is recognized by the American Society for Testing Materials, ${ }^{2}$ and provisionally by the American Institute of Electrical Engineers. ${ }^{3}$ If induction and magnetizing force be looked upon as the same physical quantity, it is natural and unobjectionable to express them in the same unit. This view 
has been a consequence of the importance hitherto assigned to the magnetic pole. Thus, induction and magnetizing force may be looked upon as having the same physical nature when magnetizing force is defined as the force on a unit magnetic pole in a medium and induction as the force on a unit magnetic pole in a very short, wide cavity with its width perpendicular ${ }^{4}$ to the direction of magnetization of the medium. There are, however, objections to this conception of induction, which will be pointed out below.

The electromagnetic units were so chosen that $\mu_{\circ}$ (permeability of empty space) $=\mathrm{I}$. This simplifies certain calculations. Thus, letting $B_{\circ}=$ induction in a nonmagnetic medium, the equation $B_{\mathrm{o}}=\mu_{\mathrm{o}} H$ may be written $B_{\mathrm{o}}=H$ in electromagnetic units. Also, the equation $B=B_{\circ}+4 \pi J$, of Table $\mathrm{I}$, is often written $B=H+$ $4 \pi J$ in electromagnetic units. The numerical equality of $H$ and $B$ in nonmagnetic media, and the appearance of $B$ and $H$ as terms in the same equation, both tend to encourage the idea that they are of the same physical nature. A corollary of this idea is that permeability is a mere numerical ratio and not a physical quantity. The equations $B_{\circ}=H$ and $B=H+4 \pi J$ do not hold in units other than electromagnetic, as will be shown in Section IV.

Arguments from the dimensions of the quantities have frequently been adduced in attempts to settle this question concerning the units of $B$ and $H$. It has been maintained, on the one hand, that $B$ and $H$ are dimensionally identical in the ordinary electromagnetic system ( $\mu$ suppressed), and are therefore expressible in the same unit; and, on the other hand, that when $\mu$ is retained as one of the fundamental quantities of the electromagnetic system, $B$ and $H$ are dimensionally different and therefore can not be expressed in the same unit. The question of the "gauss" has recently been discussed from this latter standpoint in an interesting article by M. Ascoli. (See footnote I6, p. 6rg.) Such arguments are certainly not conclusive. Dimensional identity or difference tells nothing about the physical difference between two quantities. The argument that dimensional identity indicates physical identity is refuted by the example of energy and torque. Who would measure torque in joules or calories? To maintain, on the other hand, that dimensional difference proves physical difference is not conclusive, as illustrated by the very fact that the two sets of dimensions, the electrostatic and electromagnetic, apply to every electric quantity. Dimensions are matters of convenience,

4 Maxwell, Electricity and Magnetism, par. 400. 
and dimensional arguments can no more settle a question of the physical nature of a quantity than mathematical convenience can justify the physical identification of lines of magnetic force and lines of magnetic induction. Inasmuch as dimensional reasoning has been considered by various writers to be valid to decide the physical relation of $B$ and $H$, it is thought worth while to consider further in this paper the actual physical nature of the two quantities.

There are a number of reasons for considering induction and magnetizing force to be different in physical nature, just as the deflection of a spring is physically different from the mechanical force producing it. As the names of the two quantities suggest, $B$ characterizes the magnetized state of the medium and $H$ is the agency tending to produce a magnetized state. Perhaps the distinction is most clearly seen by the analogies furnished in the discussion of the magnetic circuit (preceding section). There is no confusion between $D$, the electric displacement, which characterizes a state of the medium, and $\mathcal{E}$, the electric field intensity which produces the displacement. The distinction is even clearer between $U$, the current density in a medium, and the $\mathcal{E}$ which causes it.

Another analogy which points to the physical distinctness of $B$ and $H$ and to $\mu$ as a physical property rather than a mere numerical ratio is based on the energy of the medium. The various kinds of energy are each the product of two factors. Energy may be a force $x$ a distance, a mass $x$ half the square of a velocity, a pressure $\times$ a volume, etc. The energy density of an electrified medium is proportional to $D E$, and of a magnetized medium is proportional to $\int H d B$. The energy density of a magnetized medium may be written (where $\mu$ is constant $^{5}$ ) as $\frac{\mathrm{I}}{8 \pi} \mu H^{2}$. If $\mu$ were a mere numerical ratio, the magnetic energy density would involve a "generalized" force without the corresponding "flux." This would be contrary to the usual dynamical ideas

Again, $\mu$ is one of the two quantities ( $\mu$ and $K$ ) which are effective in the propagation of electromagnetic waves. Therefore $\mu$ must be an actual physical property (for two physical properties are dynamically necessary in a medium for the propa-

5 Since $\mu$ is not constant for magnetic materials, the usual expression for the energy density is not very useful. The correct expression is in the integral form. Most reference books fail to emphasize this suff. ciently. 
gation of waves) unless $K$ involves both properties. If electricity is of dynamical nature, therefore, either $\mu$ or $K$ must be a physical quantity and both may be.

In a number of respects $B$ and $H$ are of quite a different character. At the surface of separation between two media only the normal component of $B$ is continuous, while only the tangential component of $H$ is continuous. It is possible for induction to exist in a material with no magnetizing force. An example of such a case is a permanent ring magnet with no applied $H$, in which, therefore, $H=0$ and $B$ is finite. (An interesting electrical analog of this is Kammerlingh Onnes's electrical conductor at a temperature very near the absolute zero, in which there was a current with no electromotive force.)

The point under discussion may be considered from the standpoint of convenience. While the conception of $B$ and $H$ as physically equivalent was found desirable in magnetic theories based upon magnetic poles, it is not so useful when the relations of magnetism to electricity are dealt with. While a given $H$ is associated with a given current, it is $B$ that is concerned in the phenomenon of induced electromotive force; that is, induced electromotive force depends on the medium, while current and its accompanying $H$ are independent of the medium. Since these relations are at the basis of most magnetic practice, it is just as desirable to consider $B$ and $H$ to be different quantities as electromotive force and current.

If it be agreed, in accordance with the foregoing considerations, to look upon $B$ and $H$ as physically distinct entities, the question remains: Should units of the two quantities be known by the same name? The author would answer this question in the negative. It is always awkward, and tends to confusion, when the same name is used for units of two physically different quantities. One will not be certain in all cases which quantity he is dealing with. A number of examples of this sort of confusion are readily found. An instance is the "pound," used for the unit of mass and also for the unit of force or weight in engineering. This use of "pound" as the name for both units tends to obscure the difference between mass and weight, and to complicate discussions of the variation of weight arising from the variation of the acceleration of gravity. Another example occurs in connection with the units of energy and torque. The term "kilogrammeter" might serve for the unit of either energy or torque. In order to show which is meant, however, it is considered ${ }^{6}$ worth 
while, when giving the unit of torque, to reverse the customary designation of the unit of energy, thus using "meter-kilogram" for the torque unit; or, following Kennelly, to indicate that it is a perpendicular product by the expression "kilogram perp. meter."

Nomenclature is sometimes proposed which overlooks the disadvantage of calling two different things by the same name. Thus, in the Giorgi system of units described below, it was proposed to call the unit of magnetic permeance the henry. This suggestion has not met with much favor; while permeance has the same dimensional formula as inductance, it is none the less a distinct physical quantity. The same fundamental objection applies to a proposal now being considered ${ }^{7}$ to call a unit of luminous flux the watt. Luminous flux is detected physiologically, and as long as it has a physiological aspect can not be expressed in terms of a purely physical unit.

Still another similar case is the use of "centimeter" as the name for the cgs electrostatic unit of capacity and also as the name for the cgs electromagnetic unit of inductance. Neither inductance nor capacity, of course, is physically the same as length and the name is therefore not entirely satisfactory.

Finally, the objection to using the same name for units of two or more different physical quantities may be put upon the basis of convenience. It conduces to misunderstandings to use a word in several different meanings. The difficulties are perhaps less serious after one becomes familiar with the nomenclature and understands the double meanings, but they are a serious stumblingblock to the formation of ideas by students. In practice it is better to have the units distinctively named. So long as "gauss" is used both for the unit of $B$ and of $H$, if one were told that roo gausses were used in the iron of a certain apparatus (similarly to the use of a certain number of amperes or volts in an electric circuit) he would not know unless aided by context or convention whether gausses of $B$ or of $H$ were meant. The difficulty is made worse by the fact that the name "gauss" has been used for the unit of still another quantity; it was tentatively adopted by the British association committee on electrical standards in 1895 for the cgs unit of magnetomotive force.

At the present time gauss is used more generally for the cgs unit of $B$ than in any other sense, ${ }^{8}$ and it is probably well to use

\footnotetext{
7 Ives, Phys. Rev., 5, p. 270 ; 1915.

8 The statement is frequently made that the cgs unit of $H$ is regularly called the "gauss" in terrestrial magnetic work. This is by no means true, there being no consistency of practice regarding the unit of $H$ in that field of work.
} 
it only in that way. A name that is widely used for the cgs unit of $H$ is the gilbert per centimeter, the cgs unit of $f$ being the gilbert. (This is similar to the expression of electric field intensity in volts per centimeter.) The cgs unit of $\phi$ is the maxwell. When it is desired to avoid the term "gauss" altogether, the cgs unit of $B$ is called the maxwell per square centimeter.

\section{RATIONALIZATION OF THE UNITS}

Oliver Heaviside ${ }^{9}$ pointed out in 1882 that the factor $4 \pi$ could be removed from many of the electric and magnetic equations by the use of a new set of units. He called them "rational" units, because some of the equations appeared to have a more reasonable correspondence with the underlying theory when $4 \pi$ was eliminated. Since that time there have been other proposals to define units which would change the position of $4 \pi$ in the equations. The term "rationalization" has persisted in the literature, meaning a redistribution of the $4 \pi$ 's in an advantageous manner.

\section{THE HEAVISIDE SYSTEM}

The units used by Heaviside and by a few recent writers differ by powers of $4 \pi$ from the ordinary cgs units. In these units the factor $4 \pi$ is eliminated from certain formulas, and the electric and magnetic equations are made symmetrical. These units are used as a Gaussian system; that is, electrostatic units are used for purely electric quantities and electromagnetic units for magnetic quantities. Every equation containing both electric and magnetic quantities must contain as a factor the first or second power of $c$, the number of electrostatic units in one electromagnetic unit of electric charge, which is equal to the velocity of light in space. For instance, the equations for magnetic flux become

$$
\phi=c \frac{\mathcal{L} I}{N}=c \int E d t
$$

Heaviside suggested that units of more practical magnitude could be obtained by taking $10^{8}$ times certain of his units instead of using the various multiples, $10^{9}$, $10^{8}$, $10^{-1}$, of the "practical" electromagnetic units. His suggested multiples have never come into use.

9 Electrician, 10, p. 6, I882; Electrical Papers, 1, pp. 199, 262, 432; 2, pp. 543, 575, I892. 


\section{TABLE 3}

\section{Heaviside System}

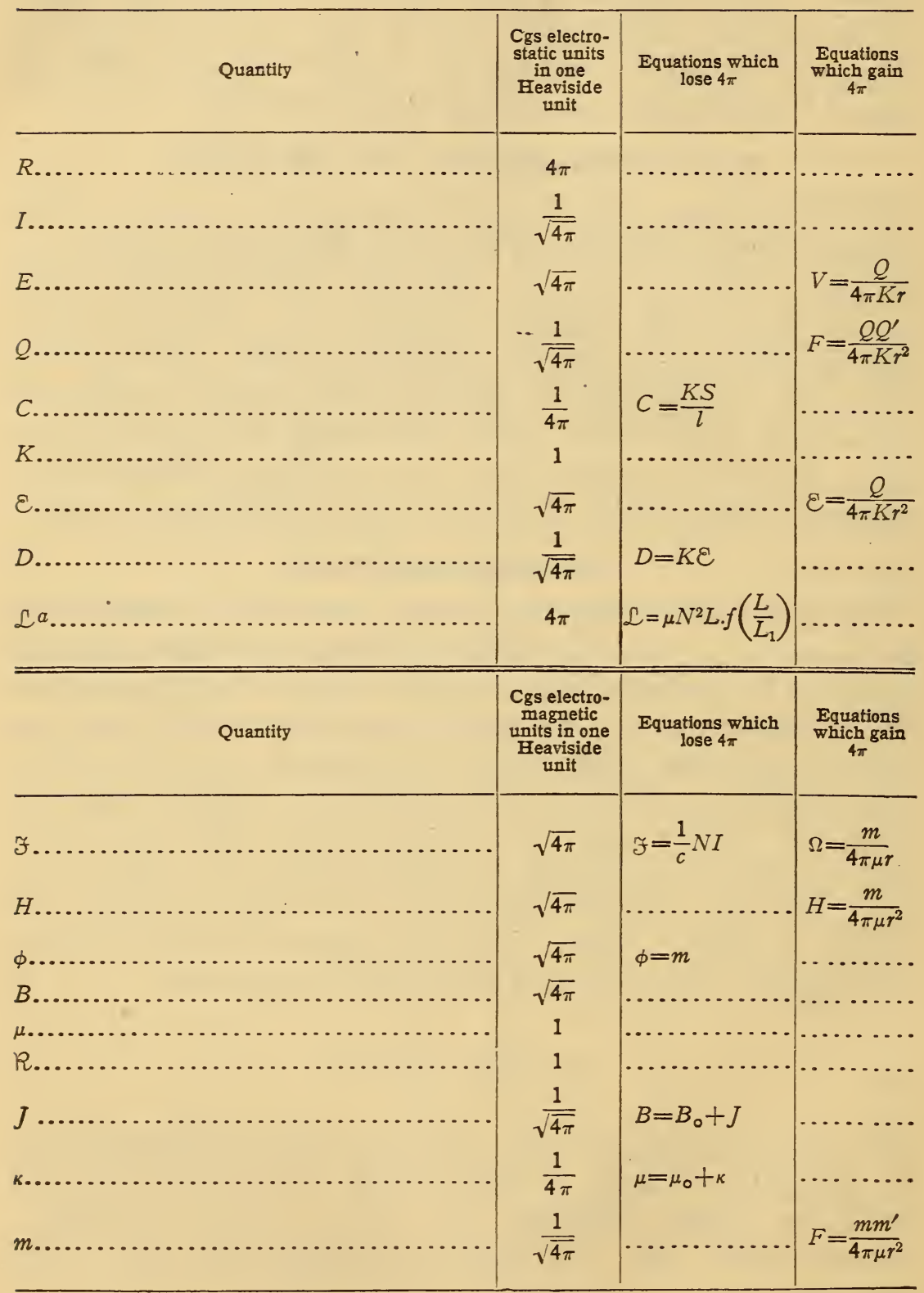

a In the formula, $L=$ radius and $L_{1}=$ length of a solenoid, and $f$ means "function of." 
Table 3 shows that the factor $4 \pi$ has been removed from every equation which contained it in the ordinary systems. The same factor has been caused to appear in certain equations which were formerly free from it. It is sometimes stated as the main objection against the Heaviside system that it merely shifts the $4 \pi$ from some formulas to others. However, the existence of $4 \pi$ in the formulas

$$
V=\frac{Q}{4 \pi K r}, F=\frac{Q Q^{\prime}}{4 \pi K r^{2}}, \text { and } \varepsilon=\frac{Q}{4 \pi K r^{2}}
$$

and the three analogous formuras in magnetism are not troublesome because these equations are seldom used in numerical computations. The $4 \pi$ is removed from the equations used in practical work, where its presence is obnoxious. Furthermore there is some theoretical justification for the $4 \pi$ in those equations which contain it when Heaviside units are used. Consider, for instance, $H=\frac{m}{4 \pi \mu r^{2}}$. If we define the unit pole so that unit flux emanates from it, we should have, since $B=\frac{m}{S}$, for a spherical surface surrounding $m$ at the distance $r, B=\frac{m}{4 \pi r^{2}}$. From $B=\mu H$, it follows that $H=\frac{m}{4 \pi \mu r^{2}}$; that is, $4 \pi r^{2}$ suggests the circumscribing sphere.

The $4 \pi$ is not only removed from $\tilde{F}=4 \pi N I$, but also from curl $H=4 \pi U$ and other forms of the First Law of Electromagetism. (The various forms of the law are given on p. 625.) Some forms of the law do not contain $4 \pi$ in the ordinary units, but do in Heaviside units, as follows:

$$
\begin{aligned}
\Omega & =I \frac{\omega}{4 c}(\omega=\text { solid angle }) \\
d H & =\frac{I d l}{4 \pi c r^{2}} \sin (r, d l) \\
H & =\frac{I}{2 \pi c r}, \text { for an infinitely long, straight conductor. }
\end{aligned}
$$

Many formulas for electrostatic capacity are more convenient in Heaviside than in ordinary units. The capacity of a sphere, however, is $K r$ in ordinary units, and $4 \pi K r$ in Heaviside units. It is desirable to have the $4 \pi$, which originates in the properties 
of a sphere, appear in the formulas pertaining to spheres rather than elsewhere. It is a practical convenience, also, to have the awkward numerical coefficient in the formula for a sphere rather than in the formula for a plate condenser, $C=\frac{K S}{l}$, since the latter is the only case that is frequently met with in practice.

In the Heaviside system the factor $4 \pi$ is not only removed from all the formulas where it occurs in the ordinary systems to more appropriate places in the theory, but the formulas of electrostatics and magnetism are also made identical in form. The symmetry and convenience of the system have aroused admiration in many who have studied the electric units. It was seriously considered for general adoption in England in IS95 and again in 1905. The enormous difficulties of changing the magnitudes of practically all electric units and standards stood in the way and will render the change more and more impossible as time goes on. Some writers ${ }^{10}$ on the theory of electricity use these units at the present time, leaving it to their readers to introduce conversion factors when applying the theory to measurements. This makes the reading of their works much more difficult for the majority of students and electrical workers. As already shown, the $4 \pi$ necessarily appears in some places, so that the reader is not freed from considering it but must deal with it in a way entirely unfamiliar to him. The removal of this factor from certain equations is furthermore not necessarily an advantage, as will be shown below (p. 626).

\section{OTHER ATTEMPTS TO ELIMINATE $4 \pi$}

The demand for a rationalization of the units has increased as electric waves have come into prominence, requiring the simultaneous use of more parts of electromagnetic theory than other phenomena require. In I900 the American Institute of Electrical Engineers adopted a recommendation to bring up the matter at the next international electrical congress.

There have been a number of attempts to evade the $4 \pi$ difficulty without recourse to the Heaviside system, which would require such an uprooting of all existing units and standards in electricity. The first proposal was that of Prof. John Perry in I889, and all the subsequent proposals have been variations of Perry's. He was requested by the British association electrical standards commit-

${ }^{10} \mathrm{H}$. A. Lorentz, The Theory of Electrons, 1909; O. W. Richardson, The Electron Theory of Matter, I914, 
tee to prepare a report on magnetic units and published his proposal ${ }^{11}$ in $\mathrm{r} 89 \mathrm{I}$. The idea was to define a new unit of permeability so as to make $\mu_{\circ}=4 \pi \times 10^{-8}$. The unit of $\mathfrak{F}$ was to be the ampereturn, of $H$ the ampere-turn per centimeter, and of $\phi$ and $B$ 10 8 times the cgs electromagnetic units. These units permitted the retaining of the equation $B=\mu H$, unchanged in form. They also gave the following simplified equations:

$$
\begin{aligned}
\mathfrak{F} & =N I \text { ( } I \text { being in amperes). } \\
\frac{d \phi}{d t} & =E \text { (volts). }
\end{aligned}
$$

The $4 \pi$ is eliminated from the first of these equations and the factor $10^{8}$ from the second. These units do not furnish a satisfactory solution to the difficulty because of the change in the value of $\mu$. The convenience of having $\mu=\mathrm{I}$ for the ether or for air has been too great to be sacrificed.

Since Perry's proposal, the same or nearly the same idea has been suggested by several different persons. F. G. Baily ${ }^{12}$ made the identical suggestion in $\mathrm{I} 895$. In $1900 \mathrm{R}$. A. Fessenden ${ }^{13}$ proposed that $\mu_{\mathrm{o}}$ be taken $=4 \pi$, and that similarly a new unit for dielectric constant be defined such that $K_{\mathrm{o}}=\frac{\mathrm{I}}{4 \pi}$. The unit of $\phi$ was to be the cgs electromagnetic unit, and the unit of $r$ Io ampereturns. This proposal redistributed the $4 \pi$ 's in the electric and magnetic equations just as the Heaviside units do. Objections to giving $\mu_{\mathrm{o}}$ and $K_{\mathrm{o}}$ values different from $\mathrm{I}$ has prevented acceptance of this system, just as in the case of the Perry units.

The system proposed by Fessenden involved the abandonment of the ohm, ampere, and other "practical" units and the use of cgs electromagnetic units instead. A modification of Fessenden's system was suggested by J. A. Fleming. ${ }^{14}$ The value of $\mu_{0}$ was taken $=4 \pi$, as in Fessenden's system, and the "practical" multiples of the cgs units suggested by Heaviside were used; that is, the unit of resistance was to be $10^{8} \mathrm{cgs}$ electromagnetic units, the unit of current I cgs unit, etc. The single multiplier Io $^{8}$ was to be used. The advantage of these proposed units is slight com-

11 Electrician, 27, p. 355; 189.

12 Electrician, 35, p. 449; 1895.

13 Elec. World, 35, p. 282, I900; Phys. Rev., 10, pp. I, 83, 1900.

14 Electrician, 44, pp. 324, 366, 402; 1900. 
pared with the inconvenience of abandoning those at present in use.

It was shown by G. Giorgi ${ }^{15}$ in I90I that an entire system could be built up about the idea of Perry retaining the ordinary units such as the ohm and the volt. The same system was proposed a little later by D. Robertson. Giorgi called it the "absolute practical" system, because it is a single coherent system utilizing the "practical" electric units. The system has a unique dimensional basis, the fundamental quantities being length, mass, time, and electric resistance. As the units of these he chose the meter, the kilogram, the second, and the international ohm. This system is rationalized-that is, the $4 \pi$ 's are redistributed-exactly as in the Heaviside and the Fessenden systems. There is a very complete correspondence of the electric and magnetic equations. Because of the symmetry and consistency of the system, Giorgi was led to give the same name to units having the same dimensions; for example, henry for inductance and for permeance, ampere for current and for magnetomotive force. He would also express both energy and torque in joules. Such terminology is misleading, as pointed out above; ampere-turn is a much better name for the unit of magnetomotive force, reserving ampere for current.

Many of the units are those at present in use. A few, like the coulomb per square meter and the ampere-turn per meter, are not used. The weber is not used, being too large a unit for the requirements of practice. The units of $K$ and $\mu$ are, of course, the least satisfactory. They are so chosen that $K_{0}=8.842 \times 10^{-12}$ and $\mu_{\mathrm{o}}=\mathrm{I} .2566 \times 1 \mathrm{I}^{-6}$. The Heaviside system is preferable in this respect, since it makes $K_{0}=\mathrm{I}$ and $\mu_{0}=\mathrm{I}$. The superiority of the Giorgi system is its retention of the ordinary "practical" units for the principal electric quantities. Its equations also are slightly more simple in form than the Heaviside equations, since the factor $c$ is not required in $\mathcal{F}=N I$ and other equations involving both electric and magnetic quantities. In its completeness and consistency the Giorgi system is admirable. Its advantages, however, largely depend on acceptance of the system as a whole, and the requirements of practice always work against the consistent use of a single system of units. In practice, convenient decimal multiples are used. 
TABLE 4

Giorgi System

\begin{tabular}{|c|c|c|c|}
\hline Quantity & Unit & $\begin{array}{c}\text { Dimensional } \\
\text { formula }\end{array}$ & $\begin{array}{l}\text { Cgs electromag- } \\
\text { netic units in one } \\
\text { Giorgi unit }\end{array}$ \\
\hline$R \ldots .$. & Ohm............ & {$[R]$} & $10^{9}$ \\
\hline$I \ldots \ldots$ & Ampere......... & {$\left[\frac{L M^{1 / 2}}{T^{3 / 2} R^{1 / 2}}\right]$} & $10^{-1}$ \\
\hline$E \ldots$ & Volt........... & {$\left[\frac{L M^{1 / 2} R^{1 / 2}}{T^{3 / 2}}\right]$} & $10^{8}$ \\
\hline$Q \ldots$ & Coulomb.......... & {$\left[\frac{L M^{1 / 2}}{T^{1 / 2} R^{1 / 2}}\right]$} & $10^{-1}$ \\
\hline$C \ldots$ & Farad .............. & {$\left[\frac{T}{R}\right]$} & $10^{-9}$ \\
\hline$K \ldots$ & Farad per meter... & {$\left[\frac{T}{L R}\right]$} & $\frac{4 \pi}{10^{11}}$ \\
\hline$\varepsilon$ & Volt per meter.... & {$\left[\frac{M^{1 / 2} R^{1 / 2}}{T^{3 / 2}}\right]$} & $10^{6}$ \\
\hline D.... & Coulomb per $\mathrm{m}^{2}$. & {$\left[\frac{M^{1 / 2}}{L T^{1 / 2 R^{1 / 2}}}\right]$} & $10^{-5}$ \\
\hline $\mathcal{L} \ldots$ & Elenry.......... & {$[T R]$} & $10^{9}$ \\
\hline F. & Ampere......... & {$\left[\frac{L M^{1 / 2}}{T^{3 / 2} R^{1 / 2}}\right]$} & $\frac{4 \pi}{10}$ \\
\hline$H$. & Ampere per meter & {$\left[\frac{M^{1 / 3}}{T^{3 / 2} R^{1 / 2}}\right]$} & $\frac{4 \pi}{10^{3}}$ \\
\hline$\phi \ldots$ & Weber........... & {$\left[\frac{L M^{1 / 2} R^{1 / 2}}{T^{1 / 2}}\right]$} & $10^{8}$ \\
\hline B.. & Weber per $\mathrm{m}^{2} \ldots$ & {$\left[\frac{M^{1 / 2} R^{1 / 2}}{L T^{1 / 2}}\right]$} & $10^{4}$ \\
\hline & Henry per meter.. & {$\left[\frac{T R}{L}\right]$} & $\frac{10^{7}}{4 \pi}$ \\
\hline$R$. & $1 /$ henry.......... & {$\left[\frac{1}{T R}\right]$} & $\frac{4 \pi}{10^{9}}$ \\
\hline$J \cdot$. & Weber per $\mathrm{m}^{2} \ldots$ & {$\left[\frac{M^{1 / 2} R^{1 / 2}}{L T^{1 / 2}}\right]$} & $\frac{10^{4}}{4 \pi}$ \\
\hline & Henry per meter.. & {$\left[\frac{T R}{L}\right]$} & $\frac{10^{7}}{(4 \pi)^{2}}$ \\
\hline$m \ldots$ & Weber.... & {$\left[\frac{L M^{1 / 2} R^{1 / 2}}{T^{1 / 2}}\right]$} & $\frac{10^{8}}{4 \pi}$ \\
\hline
\end{tabular}

M. Ascoli has recently shown ${ }^{16}$ that it is possible to frame a system which retains the ordinary "practical" units for certain quantities, but is based on the meter, kilogram, second, and $\mu_{0}=\mathrm{I}$;

${ }^{16}$ L'Elettrotecnica, 2, p. 73r, 1915. (Translation by Kennelly in Elec. World, 67, p. 876, 1916.) 
by inserting a numerical factor $\lambda$ in the equation $F=\frac{2 m I}{\lambda l}$ ( $F$ is the force on a magnetic pole $m$ at distance $l$ from the infinitely long, straight current $I$ ). He states that this quantity $\lambda$ is a fifth fundamental quantity in a system of electric and magnetic units. This does not seem justified, for $\lambda$ is not a physical quantity or property. It is merely a numerical constant, of the same standing as a constant a would be in the relation between current and quantity of electricity, thus, $I=\frac{Q}{a t}$. It does not lead to any useful result to give $\lambda$ a value different from unity. In the system which Ascoli proposes (not, of course, for practical use), $\lambda=10^{7 / 2}=3162$. The magnetic units have peculiar magnitudes in this system. The unit of $H$, for instance, is $\sqrt{\text { IO }}$ cgs electromagnetic units. Since this system gives new units of incommensurable values and makes certain equations more complicated, there does not seem to be any justification for the unusual assumption on which it is based. This system does not affect the $4 \pi$ situation at all; it is like all the other systems discussed in this section merely in that it modifies the accepted equations and units.

The only writer at the present time (so far as the author has found) who uses units such that $K_{0}$ and $\mu_{0}$ contain the factor $4 \pi$ is Prof. V. Karapetoff. He makes use of the international ohm and ampere as fundamental units, uses the ampere-turn for $\mathcal{F}$ and the cgs units for $\phi$ and $B$. In building up the system of units, he removes the factor $4 \pi$ from certain defining equations, resulting in new units of $K$ and $\mu$. The relation of these units to $4 \pi$ is regarded by Prof. Karapetofi as purely an incidental or historical matter. This system has a commendable simplicity. It involves few changes from the ordinary units. The $4 \pi$ 's, of course, require caution, as their removal from certain equations means their reappearance in certain others. This system gives the most logical basis for magnetic units of the Perry type $\left(\mu_{0}\right.$ and $K_{0}$ different from unity), in that it takes the international ohm and ampere as starting-points in the system instead of $\mu$ or $K$.

All of these proposals to eliminate $4 \pi$ without recourse to the Heaviside system have involved the incorporation of $4 \pi$ in the value of $\mu$. The fundamental features of the various systems are compared in the following table: 
TABLE 5

Proposals Regarding Magnetic Units

\begin{tabular}{|c|c|c|c|c|}
\hline $\begin{array}{c}\text { Author of } \\
\text { proposal }\end{array}$ & $\mu_{0}$ & $\begin{array}{l}\text { Unit of magneto- } \\
\text { motive force }\end{array}$ & Unit of flux & Remarks \\
\hline Perry........ & $4 \pi \times 10^{-9} \ldots$ & Ampere-turn...... & $10^{5} \mathrm{cgs}$ electromagnetic & \\
\hline Fessenden... & $4 \pi \ldots \ldots$ & 10 ampere-turns.. & $1 \mathrm{cgs}$ electromagnetic & $\left\{\begin{array}{l}K_{0}=\frac{1}{4 \pi} \text { electrostatic. } \\
\text { Cgs system rationalized. }\end{array}\right.$ \\
\hline Fleming.... & $4 \pi \ldots$ & .....do.. & ..... do. & $\left\{\begin{array}{l}\text { Unit of } R=10^{8} \mathrm{cgs} \\
\text { Unit of } I=1 \mathrm{cgs}\end{array}\right.$ \\
\hline Giorgi..... & $4 \pi \times 10^{-7} \ldots$ & Ampere-turn...... & $10^{8} \mathrm{cgs}$ electromagnetic & $\left\{\begin{array}{l}K_{\circ}=8.842 \times 10^{-12} \text { electrostatic. } \\
\text { "Practical"system rationalized. }\end{array}\right.$ \\
\hline Karapetoff $^{a}$ : & $\frac{4 \pi}{10}$. & & $1 \mathrm{cgs}$ electromagnetic.. & $\left\{K_{\circ}=\frac{1}{4 \pi}\right.$ electrostatic. \\
\hline
\end{tabular}

$a$ The values of $\mu_{0}$ and $K_{\mathrm{o}}$ are not fundamental, but derived, in the Karapetoff system. They result from the use of the ampere and ohm as fundamental units together with the elimination of $4 \pi$ from certain equations.

\section{THE TWO SETS OF MAGNETIC UNITS}

As pointed out at the beginning of Section III, two sets of magnetic units are actually in use, both being derived from the international electric units. The point of departure between the two is magnetomotive force, the unit being the gilbert in one set of units and the ampere-turn in the other. The ampere-turn is in widespread use, particularly among those who work with magnetic apparatus. It has usually been regarded as an isolated unit rather than a member of a system. It will be shown here that its use leads very simply to a complete set of magnetic units, which are furthermore rationalized at least as satisfactorily as the Heaviside units.

The ampere-turn is a very convenient unit of $\mathrm{mmf}$, the $\mathrm{mmf}$ in a magnetic circuit being equal to the product of the number of amperes by the number of turns of the electric circuit linking the magnetic circuit. The magnetic potential difference along a part of the magnetic circuit can be expressed in ampere-turns also, just as volts can be used to express the electric potential difference between two parts of a circuit as well as the total emf induced in the circuit. The ampere-turn is equal to $\frac{4 \pi}{\mathrm{IO}}$ gilberts, and both may be used for mmf's or for mpd's produced otherwise than by electric currents, just as the "pound" is used as a unit for forces produced otherwise than by gravity.

From the ampere-turn is derived the ampere-turn per centimeter as the unit of $H$. This is a very useful unit, but the name 
"ampere-turn per centimeter" may be misleading; a new name would be desirable. $H$ inside a solenoid is not equal to the product of the number of amperes by the number of turns per centimeter of winding, except in the case of a torus or a very long, single-layer solenoid. The "per centimeter" in the name of this unit does not apply to "turn" but to "ampere-turn"; that is, in a magnetic circuit, $H$ at any point is the magnetomotive force per unit distance along the magnetic circuit at that point. The unit of $\mathrm{mmf}$ being the ampere-turn, the unit of $H$ is thus the ampere-turn per centimeter of the magnetic circuit. Prof. Karapetoff has suggested the name" atcen" as a convenient abbreviation for ampereturn per centimeter. A possible objection to this term is that its formation suggests a product (as in such words as foot-pound) instead of a quotient, which the "per" indicates. It has, however, the advantage of brevity, and is readily understood.

Table 6 gives the two sets of magnetic units. The relations involving the ampere-turn are here extended to form a complete

\section{TABLE 6}

The Two Sets of Magnetic Units

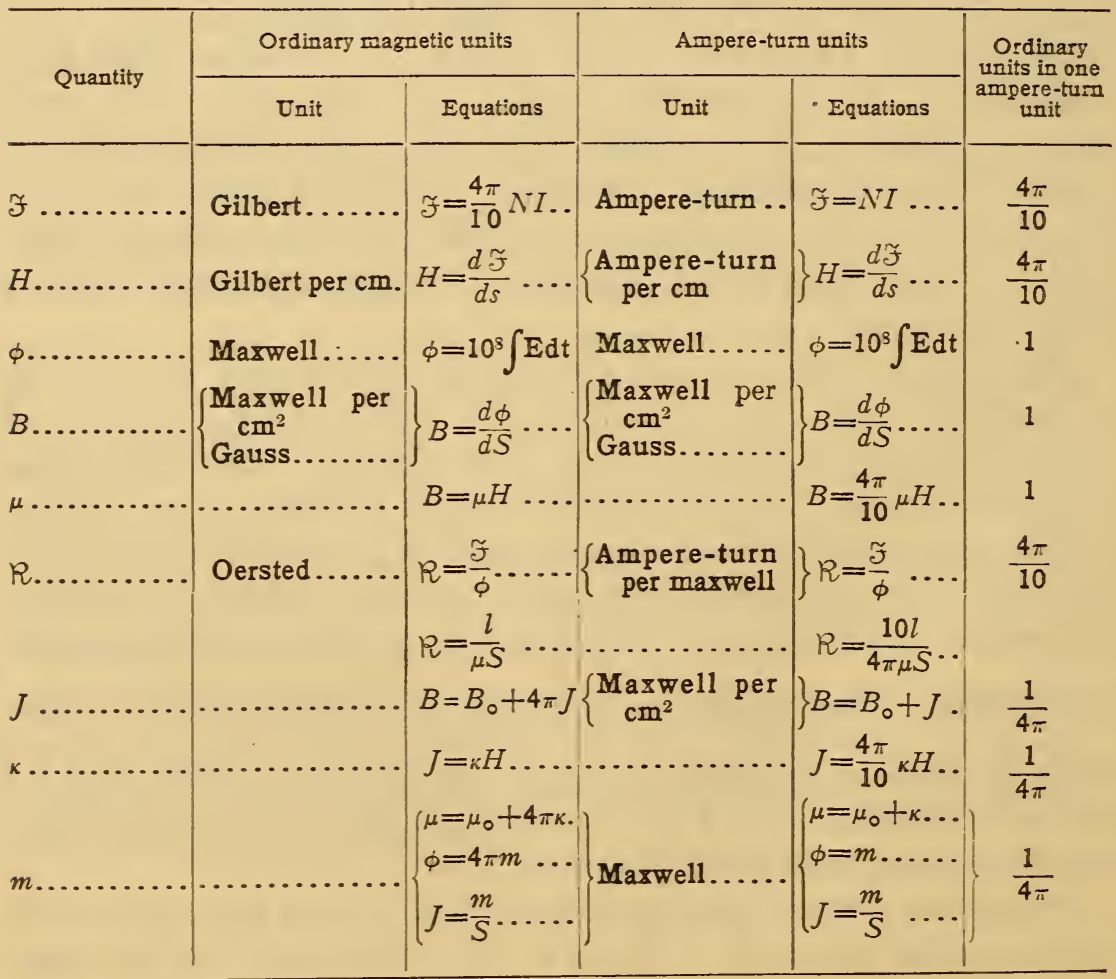


system, alternative to the ordinary magnetic units. The table is a supplement to Table $I$; the equations are based on the four fundamental units, the international ohm, international ampere, centimeter, and second. In addition to these magnetic units, a few isolated units are also in use, employing the inch instead of the centimeter.

\section{THE AMPERE-TURN UNITS}

The units of the second set in Table 6 are similar to those of Perry, Fessenden, and Karapetoff, with the very important difference that $\mu_{0}=I$, and thus no new or strange units are introduced. The only novelty is the presentation of the equations required to make the ampere-turn fit in with the units of other magnetic quantities. It fortunately turns out that these equations contain the factor $4 \pi$ in appropriate places. In a sense, the system is more conveniently "rationalized" than the Heaviside system.

The use of the same unit for magnetic pole strength and for flux is not common, because the two quantities are not equal numerically in the ordinary units. It has, however, been suggested by the authors of systems in which the units are rationalized, as the two quantities have the same physical nature. In analogous fashion, dielectric displacement and electric charge density are physically similar, and the same unit is used for both. The use of the maxwell as the unit of $m$ leads to the important consequence that $J$ is expressed in the same unit as the physically similar $B$. This usage results in changing the equation $B=B_{\circ}+4 \pi J$ into the simpler $B=B_{\circ}+J$. Some of the equations are thus simplified, not by introducing new units, but by using the ordinary units for quantities to which they have not commonly been applied.

In the ampere-turn units of Table 6 the $4 \pi$ has been removed from every equation which contained it when using ordinary units. It will also be noted that in the equations given in ampereturn units $4 \pi$ occurs only in the factor $\frac{4 \pi}{10}$ in connection with $\mu$ or $\kappa$. This factor in $B=\frac{4 \pi}{10} \mu H$ does not mean an addition of $4 \pi$ to calculations; it merely comes to the surface in this equation because $H$ is expressed in ampere turns, which removes the $\frac{4 \pi}{10}$ from the relation between $\xi$ and $I$. When the field is due to a $75741^{\circ}-17 \longrightarrow 8$ 
solenoid, the $4 \pi$ can not be escaped in the calculations. That it is legitimate in every way to have $4 \pi$ in the relation between $B$ and $H$ is suggested by the analogous relation in electrostatics, $D=\frac{I}{4 \pi} K \varepsilon$. Similarly, the equation for permeance, the reciprocal of reluctance, $\frac{I}{R}=\frac{4 \pi}{\mathrm{IO}} \frac{\mu S}{l}$, is analogous to the equation for capacity of a plate condenser, $C=\frac{I}{4 \pi} \frac{K S}{l}$. The fact that $4 \pi$ appears in the numerator in the magnetic relations and in the denominator in the electrostatic relations is of no physical significance, but explains the appearance of the factor $(4 \pi)^{2}$ in the equations below, pertaining to magnetic poles. The equations on the left are in ordinary magnetic units and on the right in ampere-turn units.

$$
\begin{aligned}
\Omega & =\frac{m}{\mu r} \\
\Omega & =I O^{7} \frac{W}{m}(W \text { in joules }) \\
H & =\frac{m}{\mu r^{2}} \\
m H & =F \text { (F in dynes }) \\
F & =\frac{m m^{\prime}}{\mu r^{2}}
\end{aligned}
$$

$$
\begin{aligned}
\Omega & =\frac{\text { IO }}{(4 \pi)^{2}} \frac{m}{\mu r} \\
\Omega & \left.=10^{8} \frac{W}{m} \text { ( } W \text { in joules }\right) \\
H & =\frac{\text { IO }}{(4 \pi)^{2}} \frac{m}{\mu r^{2}} \\
m H & =\text { IO } F(F \text { in dynes }) \\
F & =\frac{\mathrm{I}}{(4 \pi)^{2}} \frac{m m^{\prime}}{\mu r^{2}}
\end{aligned}
$$

The awkward factors on the right are not a source of inconvenience in calculations because these equations are of no technical importance whatever. These equations are not even essential to the theoretical development of the international system, as shown in Table I. In the Heaviside and all other rationalized systems the $4 \pi$ is removed from equations used in practice and relegated to these equations. In fact, the factor $(4 \pi)^{2}$ really occurs in the Giorgi and similar systems just as it does here, although it does not appear so because one $4 \pi$ is incorporated in the value of $\mu$. Those systems also possess an apparent superiority over the ampereturn system in eliminating the $4 \pi$ from some of the equations for calculating inductance and capacity. This apparent advantage is illusory, since the $4 \pi$ is in those systems existent in the numerical values of $\mu$ and of $K$, and so numerical calculations would have to deal with it. In the present system $\mu$ and $K$ preserve their ordinary values, and $\frac{4 \pi}{\mathrm{IO}}$ accompanies $\mu$ in the formal equations. 
The removal of $4 \pi$ from the first equation of Table 6 is of particular importance, as the same change applies to other forms of the same law. This is the first law of electromagnetism, and gives the mathematical relation between electric current and the magnetic field associated with it. Each of the forms given below is used by some writers as the expression of the essential electromagnetic relation. The equations on the left are in the ordinary magnetic units and those on the right in ampere-turn units. In the latter units $4 \pi$ has been removed from every equation which contained it in ordinary units and has been put into certain others.

$$
\begin{aligned}
& \mathcal{F}=\frac{4 \pi}{10} N I \\
& \Omega=\frac{I \omega}{\mathrm{IO}}(\omega=\text { solid angle }) \text {. } \\
& \int_{\mathrm{s}}^{\mathrm{s}} H d s=\frac{4 \pi}{\mathrm{IO}} I \quad \text { (Integration is } \int_{\mathrm{s}}^{\mathrm{B}} H d s=I \\
& \text { around the current.) } \\
& \operatorname{cur1} H=\frac{4 \pi}{\mathrm{IO}} U \\
& \frac{\sigma}{\mu}=\frac{I}{I O} \\
& d H=\frac{I}{\mathrm{IO}} \frac{d l}{r^{2}} \sin (r, d l) \\
& H=\frac{4 \pi N I}{\text { to } L}, \begin{array}{r}
\text { inside an infi- } \\
\text { nitely long so- }
\end{array} \\
& \text { lenoid. } \\
& H=\frac{2 \pi N I}{\text { IO } r}, \text { at center of a } \text { circle of radius } r \\
& \text { with } N \text { turns. } \\
& H=\frac{2 I}{\mathrm{IO} r}, \begin{array}{l}
\text { for an infinitely } \\
\text { long straight }
\end{array} \\
& \text { conductor. } \\
& F=\frac{\mathrm{I}}{\mathrm{IO}}[I l \times B] \\
& \phi=10^{8} \frac{\mathcal{L} I}{N} \\
& \mathcal{F}=N I \\
& \Omega=\frac{I \omega}{4 \pi} \\
& \text { curl } H=U \\
& \frac{\sigma}{\mu}=\frac{4 \pi}{\mathrm{IO}} I \\
& d H=\frac{I}{4 \pi} \frac{d l}{r^{2}} \sin (r, d l) \\
& H=\frac{N I}{L} \\
& H=\frac{N I}{2 r} \\
& H=\frac{I}{2 \pi r} \\
& F=\frac{\mathrm{I}}{\mathrm{IO}}[\mathrm{Il} \times B] \\
& \phi=10^{8} \frac{\mathscr{L} I}{N}
\end{aligned}
$$


Perhaps the most striking difference between this and previous systems of units is the shift of $4 \pi$ in the equations

$$
\begin{aligned}
& B=\frac{4 \pi}{\mathrm{IO}} \mu H \\
& \phi=m
\end{aligned}
$$

These equations are thus made more exactly analogous to the electric equations

$$
\begin{aligned}
& D=\frac{I}{4 \pi} K \varepsilon \\
& \iint D d S=Q
\end{aligned}
$$

It is perfectly legitimate and indeed preferable to have the $4 \pi$ in the first equation of each pair, $B$ and $H$ being physically different quantities (likewise $D$ and $\varepsilon$ ); and to have it removed from the second equation of each pair since the quantities on both sides of the equation are physically similar. Thus, $B$ and $H$ are not numerically equal in nonmagnetic media, and there is little tendency to regard them as physically the same quantity and measurable in the same unit. There is not much likelihood that a single name will be used to mean both maxwell per square"centimeter and ampere-turn per centimeter, as "gauss" has been used for the ordinary units of $B$ and $H$. The $4 \pi$ in the relation between $D$ and $E$ has similarly assisted in preventing confusion over their physical nature.

In Heaviside units the $4 \pi$ is removed from all the equations (I) to (4). This makes electric displacement in a vacuum numerically equal to electric field intensity. These two quantities may then be confused in their physical meanings, precisely as magnetic induction and magnetizing force have become confused as a result of their numerical equality in ordinary units. It is, of course, legitimate and is mathematically convenient to make two different physical quantities have the same numerical value, but it is often misleading and troublesome. It would therefore appear that in the ampere-turn equations the $4 \pi$ 's are more advantageousiy distributed than in the Heaviside system.

It is interesting to note that in the fourth edition (I9II) of his Theorie der Elektrizität, M. Abraham effects a slight rationaliza- 
tion of units by defining a new unit of dielectric displacement by

$$
D=K \varepsilon
$$

This leads. to

$$
\iint D d S=4 \pi Q
$$

These are symmetrical with the two magnetic equations in ordinary units:

$$
\begin{aligned}
& B=\mu H \\
& \phi=4 \pi m
\end{aligned}
$$

The units were defined in this same way by A. G. Webster in his book, The Theory of Electricity and Magnetism (r897). He, however, gave the name "induction" to the quantity defined by $K \varepsilon$. According to the view expressed above, these units put the $4 \pi$ in the least favorable place, both in the electric and the magnetic equations. Abraham mentions as an advantage of these units that they take the $4 \pi$ out of the usual relation, curl $H=4 \pi \frac{d D}{d t}$, making it

$$
\text { curl } H=\frac{d D}{d t},
$$

which is thus symmetrical with

$$
\operatorname{curl} \varepsilon=-\frac{d B}{d t}
$$

This advantage, however, may also be credited to the ampere-turn units.

The characteristics of iron are very commonly shown by means of curves in which the ordinates are $B$ in gausses, and the abscissas are $H$ either in gilberts per centimeter or in ampere-turns per centimeter. A distinction must be observed in interpreting the curves in the two cases. When the abscissas are in gilberts per centimeter, the ratio of ordinate to abscissa for any point on the curve is equal to $\mu$, the permeability. When ampere-turns per centimeter are used, $\mu$ is not equal to the ratio of ordinate to abscissa, but is $\frac{\text { IO }}{4 \pi}$ times that ratio. 
The information actually wanted for iron is sometimes the ratio of $B$ to $B_{0}$ ( $B$ o being the induction in a nonmagnetic substance with the same $H$ ) rather than the ratio of $B$ to $H$. This would apply to transformer iron, for example. Consequently, magnetic curves may be plotted with both ordinates and abscissas in gausses. The ratio of ordinate to abscissa for any point equals the permeability, entirely independently of any choice of $H$ units. This follows because $\frac{B}{B_{0}}=\frac{\mu}{\mu_{0}}$, and $\mu_{0}=\mathrm{I}$.

This discussion of the ampere-turn units is presented purely for the information of those interested in units. It is not intended to suggest that the Bureau will discard the gilbert in favor of the ampere-turn in its own work. Both sets of magnetic units have been found useful, and both will doubtless continue in use.

\section{SUMMARY}

The electric units ordinarily used are based upon certain standards defined by international congresses. These units may be considered to form a distinct system. They have been called international units to distinguish them from the corresponding units in the electromagnetic system, of which they are the representatives for the purposes of electrical measurements. The international and the electromagnetic units differ by exceedingly small amounts, and the small differences are determined by absolute electrical measurements made from time to time. The international system takes the international ohm, the international ampere, the centimeter, and the second as fundamental units. The unit of magnetic pole strength is relegated to a subordinate position, corresponding with its unimportance in practical work. The dimensional expressions of electric and magnetic units in this system have a simplicity and direct applicability lacking in other systems. The system has, however, no such simplicity or utility in the domain of mechanics nor anywhere except in electricity and magnetism. It in no way tends to supplant the classical system based on length, mass, and time.

Magnetic units have been in a somewhat confused state. The use of the word "gauss" as the name for both the unit of induction and the unit of magnetizing force is one difficulty. The international system furnishes no justification for this double usage. The two quantities hare sometimes been looked upon as physically 
the same. There are reasons, however, for considering them to have an essentially different nature, induction corresponding to the magnetized state of the medium, and magnetizing force being the agency tending to produce that state. The objections to using the same name for units of different physical quantities have been presented. The double use of "gauss" is an inconvenience in practice.

There have been various proposals from time to time to rationalize the electric and magnetic units; that is, to use units such as to redistribute the factor $4 \pi$ in the equations. In no system is this factor entirely eliminated. The first proposal, that of Heaviside, had much to recommend it and his units are used in some recent theoretical works. However, all the units are different by some power of $4 \pi$ from the ordinary units, and their general adoption now seems out of the question. Proposals since Heaviside's for the rationalization of the units have involved less change of existing units but have all had the disadvantage of incorporating $4 \pi$ in the values of the permeability and dielectric constant of empty space.

A very satisfactory rationalization is effected by the use of the ampere-turn as the unit of magnetomotive force, without recourse to any new units. There are two sets of magnetic units in use, based upon the international electric units. In one set the units correspond in magnitude to the ordinary cgs electromagnetic units. In the other, the ampere-turn and derived units are used. The equations required to make the ampere-turn fit in with the units of other magnetic quantities have been given herein. For want of a better name, the units of this set are called ampere-turn units. It has been pointed out that the ampereturn units are rationalized more nearly like the Heaviside units than any of the systems which have been proposed, in that $\mu_{\circ}$ retains its value unity. From one standpoint the $4 \pi$ 's are even better distributed than in the Heaviside system. It is of interest to find that units actually in use constitute a system which is not at all inferior to the various proposed systems. This presentation of the ampere-turn units has been given as a contribution to the discussion of magnetic units, and in no sense as a proposal to discontinue the use of the gilbert and other more common units. 
In conclusion, this study has shown that the international system, based upon and representing the electromagnetic system, is a convenient and satisfactory system of units for the purposes of electric and magnetic measurements. Proposed changes in some or all of the units do not appear to offer advantages such as to justify the confusion and inconvenience of changing the units as ordinarily used.

Washington, May 22, igi6. 


\section{APPENDIX}

\section{SYMBOLS USED}

$B=$ magnetic induction.

$C=$ electrostatic capacity.

$Q=$ quantity of electricity.

$R=$ resistance.

$c=$ velocity of light.

$D=$ electric displacement.

$E=$ electromotive force.

$\varepsilon=$ electric field intensity.

$F=$ force.

$\mathcal{F}=$ magnetomotive force.

$H=$ magnetizing force.

$I=$ current.

$J=$ intensity of magnetization.

$K=$ dielectric constant.

$K_{\mathrm{o}}=$ dielectric constant of space.

$L=l=$ length .

$\mathcal{L}=L=$ self-inductance.

$M=$ mass.

$m=M=$ mutual inductance.

$m=$ magnetic pole strength.

$N=$ number of turns.

$P=$ power.

$R=$ reluctance.

$r=$ distance from a point.

$S=$ area

$s=$ length along a path.

$T=t=$ time.

$U=$ current density.

$V=$ electric potential.

$W=$ energy.

$Z=$ impedance.

$\gamma=$ conductivity .

$\delta=$ mass resistivity.

$\kappa=$ magnetic susceptibility.

$\mu=$ permeability.

$\mu_{\circ}=$ permeability of space.

$\rho=$ volume resistivity.

$\sigma=$ strength of a magnetic shell.

$\phi=$ magnetic flux.

$\Omega=$ magnetic potential. 